\title{
A Quantitative Fluoroscopic Comparison of the Coronary Sinus Ostium in Patients With and Without AV Nodal Reentrant Tachycardia
}

\author{
JOHN D. HUMMEL, M.D., S. ADAM STRICKBERGER, M.D., \\ K. CHING MAN, D.O., EMILE DAOUD, M.D., MARK NIEBAUER, M.D., \\ and FRED MORADY, M.D.

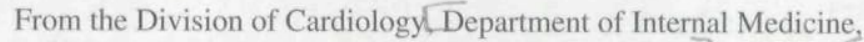 \\ University of Michigan Medical Center, Ann Arbor, Michigan [, USA]
}

Coronary Sinus Ostium. Introduction: The purpose of this study was to perform a quantitative fluoroscopic analysis of the coronary sinus ostium and its relationship to the His bundle in patients with and without AV nodal reentrant tachycardia. Sites of slow pathway ablation are often near the coronary sinus ostium, which can be located within a few millimeters of the His bundle. Whether such close proximity of the coronary sinus ostium to the His bundle is unique to patients with AV nodal reentrant tachycardia is unknown.

Methods and Results: Fifty consecutive patients (mean age $39 \pm 14$ years) with no structural heart disease underwent electrophysiologic testing and radiofrequency ablation. The study group consisted of 28 patients with inducible AV nodal reentrant tachycardia or dual AV nodal physiology and 22 patients in the control group. A coronary sinus venogram was performed in each patient. The coronary sinus ostium was similar in size in the study group (11.4 $\pm 4.5 \mathrm{~mm})$ and in the control group $(10.5 \pm 3.6 \mathrm{~mm}, P=0.2)$. The coronary sinus ostium was funnel shaped in half of the study patients and in half of the control patients $(P=1.0)$. The mean distance from the upper lip of the coronary sinus ostium to the tip of the His bundle catheter was $9.7 \pm 5.5 \mathrm{~mm}$ in the study group and $10.4 \pm 5.1 \mathrm{~mm}$ in the control group $(P=0.7)$. The mean distance from the lower lip of the coronary sinus ostium to the tip of the His-bundle catheter in the study group was $20.1 \pm 6.1 \mathrm{~mm}$ and $19.5 \pm 5.6 \mathrm{~mm}$ in the control group $(\mathrm{P}=$ 0.7).

Conclusion: This study demonstrates a wide range of normal coronary sinus ostium diameters, morphology, and anatomic relationships with surrounding structures, with no demonstrable correlation to the presence or absence of dual AV node physiology or AV nodal reentrant tachycardia. (J Cardiovasc Electrophysiol, Vol. 6, pp. 681-686, September 1995)

coronary sinus, atrioventricular nodal reentrant tachycardia, radiofrequency ablation

\section{Introduction}

Radiofrequency ablation of AV nodal reentrant tachycardia using the slow pathway approach is accomplished in the triangle of Koch, near the coronary sinus ostium. Koch's triangle is bounded

Address for correspondence: Fred Morady, M.D., Division of Cardiology, University of Michigan Medical Center, 1500 East Medical Center Drive, Ann Arbor, MI 48109-0022. Fax: 313-9367026.

Manuscript received 14 June 1995; Accepted for publication 20 July 1995 , by the tendon of Todaro, the coronary sinus ostium, and the septal leaflet of the tricuspid valve. In the anatomic approach described by Jazayeri et al., ${ }^{1}$ the area between the His bundle and the coronary sinus ostium is divided into six sections (Fig. 1), with radiofrequency applications starting at the level of the coronary sinus ostium and progressing superiorly. However, it has been observed that in patients with $\mathrm{AV}$ nodal reentrant tachycardia, the ostium of the coronary sinus at times may be close to where a His-bundle electrogram can be recorded. ${ }^{2}$ Although there have been prior anatomic studies of the triangle of Koch, ${ }^{3,4}$ there have been 


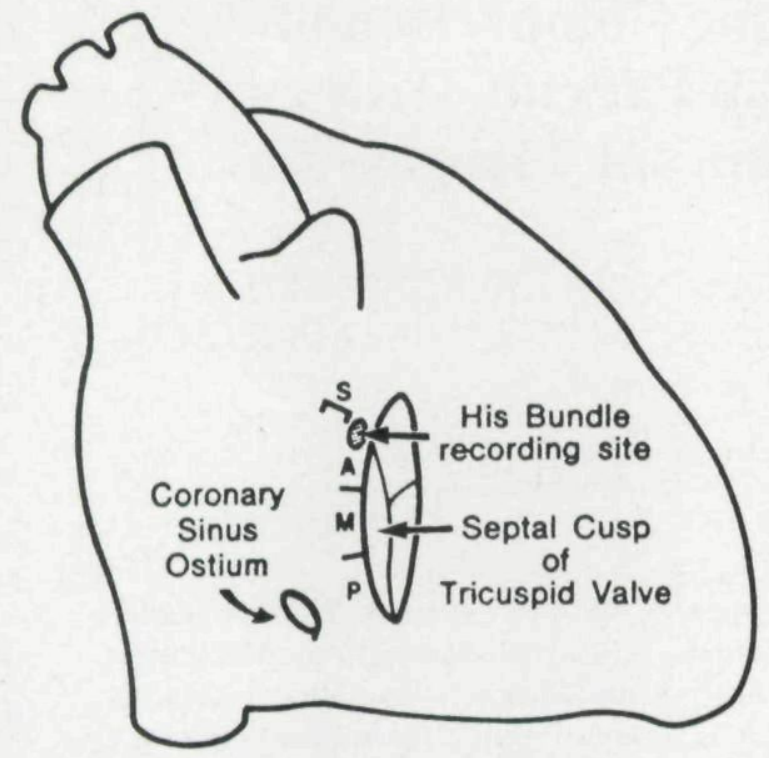

Figure 1. The six positions targeted between the coronary sinus ostium and the His-bundle catheter during slow pathway ablation. (Reproduced with permission from Jazayeri $M R$, Hempe SL, Sra JS, et al: Selective transcatheter ablation of the fast and slow pathways using radiofrequency energy in patients with atrioventricular nodal reentrant tachycardia. Circulation 1992;85:1318-1328. (c) 1992 American Heart Association.)

no prior fluoroscopic evaluations directly relevant to slow pathway ablation. Therefore, the purpose of this study was to perform a quantitative fluoroscopic analysis of the coronary sinus ostium and its relationship to the His-bundle recording site in patients with and without AV nodal reentrant tachycardia.

\section{Methods}

\section{Patients}

Fifty consecutive patients without structural heart disease undergoing an electrophysiologic evaluation for supraventricular tachycardia were enrolled in this study. There were 24 men and 26 women (mean age $39 \pm 14$ years [range 15 to 71$]$ ). All patients with inducible AV nodal reentrant tachycardia or dual AV nodal physiology were enrolled in the study group, and the remaining patients served as controls. Dual AV nodal physiology was defined as a $50-\mathrm{msec}$ or greater increase in the atrial-His interval in association with a $10-\mathrm{msec}$ decrement in the coupling interval of a single atrial extrastimulus. The study group consisted of 25 patients with inducible AV nodal reentrant tachy- cardia and 3 patients with dual AV nodal physiology who did not have inducible AV nodal reentrant tachycardia or AV nodal echo complexes. The control group consisted of 21 patients with an accessory pathway and 1 patient with paroxysmal atrial fibrillation; none of the patients in the control group had either dual AV nodal physiology or AV nodal echo complexes. Compared to the control group, the patients in the study group were older ( $43 \pm 12$ vs $35 \pm 15$ years, $P=0.04$ ), had a smaller body surface area $\left(1.8 \pm 0.2\right.$ vs $1.9 \pm 0.2 \mathrm{~m}^{2}, \mathrm{P}=$ $0.04)$, and were more likely to be women ( $76 \%$ vs $43 \%, \mathrm{P}=0.02)$.

\section{Electrophysiologic Testing}

Informed consent for the investigational protocol was obtained from all patients, and the protocol was approved by the Investigational Review Board at the University of Michigan Medical Center. Three quadripolar electrode catheters were inserted into a femoral vein and positioned in the high right atrium, across the tricuspid annulus in the His-bundle position, and at the right ventricular apex. The intracardiac electrograms and leads $\mathrm{V}_{1}, \mathrm{I}, \mathrm{II}$, and III were displayed on an oscilloscope and recorded at a paper speed of 25 to $100 \mathrm{~mm} / \mathrm{sec}$ on a Mingograf 7 recorder (Siemens-Elema, Inc., Solna, Sweden). Pacing was performed with a programmable stimulator (Bloom Associates, Reading, PA, USA). Each patient underwent overdrive pacing of the atrium and ventricle and atrial and ventricular extrastimulation to induce supraventricular tachycardia and determine its mechanism. A diagnosis of typical AV nodal reentrant tachycardia was made using standard criteria. ${ }^{5}$ If an accessory pathway was identified or if the patient had inducible AV nodal reentrant tachycardia, radiofrequency ablation was performed using previously described methods. ${ }^{6.7}$

\section{Contrast Injection}

After the clinically indicated portion of the electrophysiologic procedure, a 7-French Simmons 2. angiographic catheter (Cook Catheter, Inc., Bloomington, IN, USA) was inserted into the right atrium via a femoral vein and positioned in the coronary sinus under fluoroscopic guidance. Prior to angiography, a quadripolar electrode catheter with 2- to 5-mm interelectrode spacing was positioned to obtain the largest possible bipolar His-bundle electrogram with an atrial/ventricular electrogram ratio of 1:1 using the distal pair of electrodes. The coro- 
nary sinus was injected with radiopaque contrast ( 7 to $8 \mathrm{cc} / \mathrm{sec}$ for $3 \mathrm{sec}$ at $150 \mathrm{psi}$ ) using a power injector. Cineangiograms were recorded in the $60^{\circ}$ left anterior oblique projection.

In each patient, the borders of the coronary sinus ostium were described either as funnel shaped, with close proximity between the upper border of the coronary sinus ostium and the His bundle, or nonfunnel shaped. Examples of a funnel-shaped coronary sinus ostium and a nonfunnel-shaped coronary sinus ostium are shown in Figure 2. In assessing the morphology of the coronary sinus ostium, the intraobserver reproducibility was $86 \%$ and the interobserver reproducibility was $81 \%$.

In each patient, the maximum diameter of the coronary sinus ostium, the distance from the upper lip of the coronary sinus ostium to the His-bundle catheter, and the distance from the lower lip of the coronary sinus ostium to the His-bundle catheter were measured in a blinded fashion. Measurements were obtained in millimeters using electronic calipers, corrected for body surface area, and calibrated using the angiographic catheter width. The top of the coronary sinus ostium was determined as the superior edge of contrast intersecting a line drawn at a $90^{\circ}$ angle from the bottom of the coronary sinus at the takeoff of the inferior lip. The intraobserver reproducibility of these measurements was $88 \%$, and interobserver reproducibility was $82 \%$.

\section{Statistical Analysis}

Continuous variables are expressed as mean \pm standard deviation. Comparisons were performed using the Student's $t$-test. The correlation between the coronary sinus ostium size, distance between the upper and lower lips of the coronary sinus ostium and the His-bundle catheter, and patient age was determined by regression analysis. A P value $<0.05$ was considered significant.

\section{Results}

\section{Coronary Sinus Ostium Size}

The coronary sinus ostium was similar in diameter in the study group $(11.4 \pm 4.5 \mathrm{~mm})$ and in the control group $(10.5 \pm 3.6 \mathrm{~mm}, \mathrm{P}=0.2)$. There was no correlation between coronary sinus ostium size and age $\left(\mathrm{r}^{2}=0.10, \mathrm{P}=0.5\right)$. The diameter of the coronary sinus ostium was
$11.8 \pm 4.2 \mathrm{~mm}$ in women compared to $10.0 \pm$ $3.9 \mathrm{~mm}$ in men $(\mathrm{P}=0.1)$.

\section{Coronary Sinus Morphology}

The coronary sinus ostium was funnel shaped in $14(50 \%)$ of 28 study patients and in $11(50 \%)$ of the 22 control patients $(\mathrm{P}=1.0)$.

\section{Distance From Coronary Sinus Ostium to His- Bundle Electrogram}

In the study group, the mean distance from the upper lip of the coronary sinus ostium to the tip of the catheter in the His-bundle position was 9.7 $\pm 5.5 \mathrm{~mm}$ (range 0 to 22.6 ); in the control group the mean distance was $10.4 \pm 5.1 \mathrm{~mm}$ (range 0 to $22.8, \mathrm{P}=0.7$ ). The distance between the upper lip of the coronary sinus ostium and the His-bundle catheter was $<5 \mathrm{~mm}$ in $25 \%(7 / 28)$ of the patients in the study group, compared to $14 \%(4 / 22)$ of the patients in the control group ( $\mathrm{P}=0.3$, Fig. 3 ).

The mean distance from the lower lip of the coronary sinus ostium to the tip of the catheter in the His-bundle position in the study group was $20.1 \pm 6.1 \mathrm{~mm}$ (range 8.5 to 25.8 ); in the control group this distance was $19.5 \pm 5.6 \mathrm{~mm}$ (range 9.0 to $29.5, P=0.7$ ). The distance between the lower lip of the coronary sinus ostium and the Hisbundle catheter was $<16 \mathrm{~mm}$ in $28 \%(8 / 28)$ of patients in the study group compared to $27 \%(6 / 22)$ of the control group $(\mathrm{P}=1.0)$.

There was no correlation between the distance from the upper lip of the coronary sinus ostium to the His-bundle catheter and patient age $\left(r^{2}=\right.$ $0.17, \mathrm{P}=0.2$ ) or between the distance from the lower lip of the coronary sinus ostium to the Hisbundle catheter and patient age $\left(\mathrm{r}^{2}=0.11, \mathrm{P}=0.4\right)$.

\section{Discussion}

\section{Main Findings}

The results of this study demonstrate that there is a wide range of coronary sinus ostium diameters and morphology, with no demonstrable correlation to patient age or to the presence or absence of AV nodal reentrant tachycardia or dual AV node physiology. The study also demonstrates a large degree of variability in the distance between the upper and lower lips of the coronary sinus ostium and the location of the maximum His-bundle electrogram. In approximately $25 \%$ of patients, 

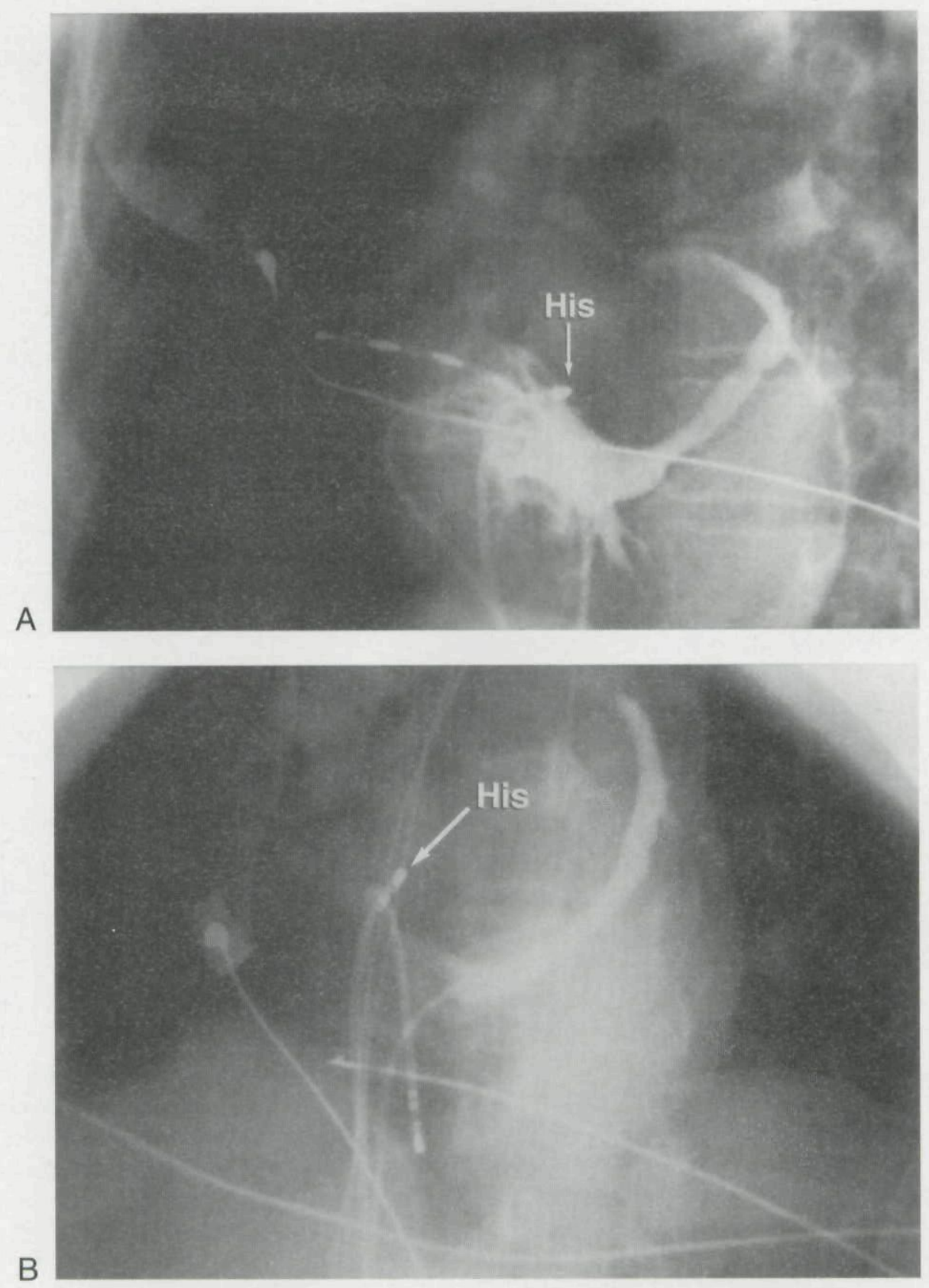

Figure 2. (A) Left anterior oblique projection demonstrates a funnel-shaped coronary sinus ostium. Note the short distance between the top of the ostium and the tip of the His-bundle catheter. (B) Left anterior oblique projection demonstrates an example of a nonfunnel-shaped coronary sinus ostium. In contrast to the funnel-shaped coronary sinus ostium depicted in panel A, this coronary sinus ostium has discrete borders. Note the greater distance between the top of the ostium and the Hisbundle catheter in comparison to that in panel $A$.

the distance between the upper lip of the coronary sinus ostium and the location of the His-bundle electrogram is $<5 \mathrm{~mm}$. The close proximity of the coronary sinus ostium to the His-bundle electrogram in some patients may explain the occasional occurrence of inadvertent AV block during slow pathway ablation.

\section{Use of Radiopaque Contrast}

A prior study recommended radiopaque contrast injection as the best method for identification of the location and boundaries of the coronary sinus ostium. ${ }^{4}$ Using this method, the coronary sinus ostium could be outlined in every patient in whom 


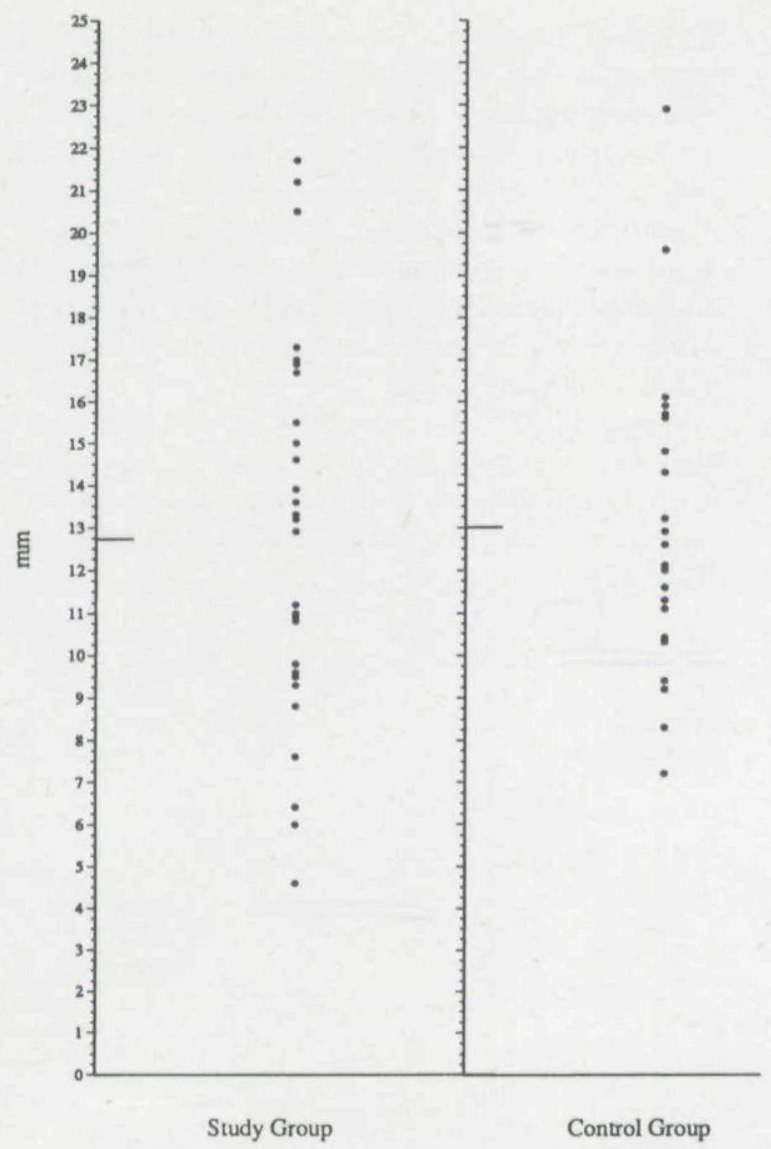

Figure 3. The range of coronary sinus ostium-His bundle catheter distances in the study group and in the control group.

contrast injection was attempted. The mean distance between the edge of the coronary sinus ostium closest to the central fibrous body and the central fibrous body was $17 \pm 3 \mathrm{~mm}$ (not corrected for body surface area) in one anatomic study. ${ }^{3}$ This measurement is similar to the mean distance of $18.5 \pm 1.2 \mathrm{~mm}$ (not corrected for body surface area) between the upper lip of the coronary sinus ostium and the His-bundle position obtained in the present study. This concordance with a prior anatomic study verifies that radiopaque contrast injection is an accurate way to delineate the boundaries of the coronary sinus ostium.

\section{Implications for Catheter Ablation}

A commonly used method for radiofrequency ablation of the AV nodal slow pathway is the anatomic approach described by Jazayeri et al. ${ }^{1}$ Using this approach, the operator systematically delivers radiofrequency applications starting pos- terior to the coronary sinus ostium (Fig. 1) in the $\mathrm{P}$ (posterior) position and moves anteriorly in a stepwise fashion toward the A (anterior) position. This method divides the region between the coronary sinus ostium and the His bundle into six radiofrequency energy application sites. The findings from contrast injection suggest that this region would compose a mean distance of approximately $13 \mathrm{~mm}$ and could be as small as $4.6 \mathrm{~mm}$, which would allow $<1 \mathrm{~mm}$ of tissue per designated radiofrequency energy application site. For this reason, strict adherence to several sites of application assigned to the region for successful slow pathway ablation may result in radiofrequency energy applications in a more anterior position than one would desire and a risk of complete AV block.

\section{Limitations}

The projection of the coronary sinus ostium was limited to a single left anterior oblique view. This may lead to an underestimation of the size of the ostium and a lack of appreciation for differences in ostial morphology. However, this single view still permits identification of the edge of the coronary sinus ostium that is nearest to the His-bundle catheter. A second limitation of the study is that the control group was almost entirely composed of patients with accessory pathways. Although posteroseptal accessory pathways may be associated with coronary sinus anomalies, ${ }^{8}$ only $4(18 \%)$ of 22 of the control patients had a posteroseptal accessory pathway, and no anomalies were noted in these patients.

\section{Conclusion}

There is great variability between patients in the distance between the coronary sinus ostium and the His-bundle electrogram. In some patients, this distance may be $<5 \mathrm{~mm}$. The relationship of the coronary sinus ostium to the His-bundle electrogram does not correlate with the presence or absence of dual AV node physiology or AV nodal reentrant tachycardia.

\section{References}

1. Jazayeri MR, Hempe SL, Sra JS, et al: Selective transcatheter ablation of the fast and slow pathways using radiofrequency energy in patients with atrioventricular nodal reentrant tachycardia. Circulation 1992;85:13181328. 
2. Mitrani RD, Klein LS, Hackett FK, et al: Radiofrequency ablation for atrioventricular node reentrant tachycardia: Comparison between fast (anterior) and slow (posterior) pathway ablation. J Am Coll Cardiol 1993;21:432-441.

3. McGuire MA, Johnson DC, Robotin M, et al: Dimensions of the triangle of Koch in humans. Am J Cardiol 1992;70:829-830.

4. Davis LM, Byth K, Lau KC, et al: Accuracy of various methods of localization of the orifice of the coronary sinus at electrophysiologic study. Am J Cardiol 1992;70:343-346.

5. Josephson ME: Clinical Cardiac Electrophysiology: Techniques and Interpretations. 2nd ed. Lea \& Febiger, Philadelphia, 1993, p. 181.
6. Calkins H, Langberg J, Sousa J, et al: Radiofrequency catheter ablation of accessory atrioventricular connections in 250 patients. Abbreviated therapeutic approach to Wolff-Parkinson-White Syndrome. Circulation 1992;85:1337-1346.

7. Kalbfleisch SJ, Strickberger SA, Williamson B, et al: Randomized comparison of anatomic and electrogram mapping approaches to ablation of the slow pathway of atrioventricular node reentrant tachycardia. J Am Coll Cardiol 1994;23:716-723.

8. Chiang CE, Chen SA, Yang CR, et al: Major coronary sinus abnormalities: Identification of occurrence and significance in radiofrequency ablation of supraventricular tachycardia. Am Heart J 1994;127: 1279-1289. 
This document is a scanned copy of a printed document. No warranty is given about the accuracy of the copy. Users should refer to the original published version of the material. 